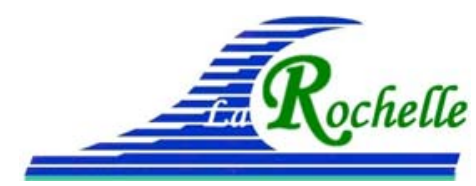

XVèmes Journées Nationales Génie Côtier - Génie Civil La Rochelle, 29 au 31 mai 2018

DOI:10.5150/jngcgc.2018.088 ～(C) Editions Paralia CFL

disponible en ligne - http://www.paralia.fr - available online

\title{
Méthodes de détermination du recul maximal des dunes côtières lors de tempêtes dans le cadre des Plans de Prévention des Risques Littoraux
}

\author{
Franck DESMAZES ${ }^{1}$, Manuel GARCIN ${ }^{1}$, \\ Alexandre NICOLAE LERMA ${ }^{2}$, Hélö̈se MULLER ${ }^{3}$
}

1. BRGM, Direction Risques et Prévention, 3 Avenue Claude Guillemin, 4500 Orléans, France.f.desmazes@brgm.fr ; m.garcin@brgm.fr

2. BRGM, Direction Régionale Nouvelle-Aquitaine, Parc Technologique Europarc

24 Avenue Léonard de Vinci 33600 Pessac, France. a.nicolaelerma@brgm.fr

3. IFREMER centre de Bretagne, DYNECO/DHYSED, ZI Pointe du Diable CS10070

29280 Plouzané, France. heloise.muller@ifremer.fr

\section{Résumé :}

Pour assurer la mise en sécurité des personnes et des biens, les risques associés à l'érosion littorale sont pris en compte par les autorités dans le cadre des Plans de Prévention des Risques Littoraux (PPRL). Pour l'aléa de recul du trait de côte, les plans visent à cartographier les secteurs d'aléa fort potentiellement affectés à une échéance de 100 ans. Dans un nouveau guide méthodologique (DGPR, 2014), la largeur de la zone d'aléa à 100 ans est évaluée à l'aide du taux de recul moyen annuel "Tx" à laquelle s'ajoute un nouveau paramètre "Lmax", correspondant à la valeur du recul du trait de côte consécutif à un évènement tempétueux majeur.

En partenariat avec la Direction Générale de la Prévention et des Risques du Ministère de la Transition Ecologique et Solidaire, le BRGM a réalisé un travail méthodologique sur la détermination de ce nouveau paramètre Lmax pour les secteurs de dunes littorales. Ce paramètre peut être évalué en utilisant plusieurs approches : (1) le recueil d'informations liées à des évènements historiques extrêmes, (2) la réalisation de mesures spécifiques réalisées "post-tempête" sur le haut de plage et le front dunaire, (3) l'utilisation de formules empiriques validées à partir d'observations terrain (méthode FEMA ; Etats-Unis) ou d'expériences en canal à houle (méthode DUROS, Pays-Bas), (4) l'utilisation de modèles morphodynamiques spécifiques (XBeach). Ce travail présente ces différentes approches d'évaluation du Lmax et différents exemples d'applications permettant une analyse critique de leur mise en œuvre.

Mots-clés :

Risques littoraux, Aléa de recul du trait de côte, Dune, Erosion, PPRL, Méthodologie. 


\section{Thème 7 - Risques côtiers}

\section{Introduction}

A la suite des pertes et dégâts catastrophiques engendrés par la tempête Xynthia (27-28 février 2010), de nombreux Plans de Prévention des Risques Littoraux (PPRL) ont été mis en œuvre ou repris entièrement au cours des dernières années pour répondre à la circulaire du 2 août 2011. La réalisation de ces PPRL "nouvelle génération" a été cadrée par la réédition du guide méthodologique relatif aux PPRL en mai 2014 (DGPR, 2014). Ce nouveau guide fourni notamment des éléments de méthode pour l'évaluation de l'aléa de submersion marine et pour l'aléa d'érosion. Pour les questions d'évaluation de la largeur de la zone d'aléa de recul du trait de côte ("Lr" équation 1), il introduit la prise en compte d'un recul maximum instantané "Lmax" pour les côtes sableuses (dunaires) en complément d'un taux de recul moyen annuel "Tx" appliqué pour 100 ans. Cet article traite plus particulièrement des méthodes d'évaluation de ce nouveau paramètre Lmax.

$L r=100 . T x+\operatorname{Lmax}$

Le guide méthodologique (DGPR, 2014) associe les forts reculs instantanés aux effets observables d'évènements tempétueux majeurs. A priori, la connaissance du paramètre Lmax passe donc essentiellement par la collecte de données d'observation. Or dans les faits, ce paramètre est actuellement mal connu et difficile à déterminer. C'est pourquoi le BRGM a réalisé depuis 2013, à la demande de la DGPR, des travaux méthodologiques sur l'estimation du paramètre Lmax pour les environnements de dunes littorales en France (DESMAZES et al., 2014a ; 2014b \& 2015). Ces travaux ont permis la rédaction d'un encart synthétique dans le nouveau guide PPRL qui mentionne trois méthodes principales : les observations et mesures, l'utilisation de formules empiriques et la modélisation numérique. La suite de cet article présente les trois types d'approches mises en œuvre et testées sur différents sites de France métropolitaine.

\section{Méthodes}

\subsection{Observations et mesures}

Les informations les plus fréquemment disponibles actuellement pour la réalisation d'un PPRL sont des données historiques qualitatives ou des estimations quantifiées mais non mesurées issues d'observations ponctuelles. Ces informations sont issues de la presse locale, d'images, de rapports d'expertise. Pour des secteurs relativement peu sensibles à l'érosion, ces éléments peuvent être suffisants pour établir le paramètre Lmax dans un PPRL, mais en fonction des sources et des détails fournis, ils peuvent être entachés d'une très forte incertitude.

Les observatoires du littoral réalisent des mesures permettant de quantifier précisément les évolutions du trait de côte et du littoral. Classiquement, il s'agit du suivi réguliers d'indicateurs de trait de côte, de profils de plage ou de levés topo-bathymétriques complets. Les protocoles d'acquisition ne sont pas forcément dédiés au suivi de l'impact 


\section{XVèmes Journées Nationales Génie Côtier - Génie Civil \\ La Rochelle, 29 au 31 mai 2018}

des tempêtes, mais l'utilisation et l'interprétation de ce type de données apparaissent indispensables pour établir un PPRL dans les secteurs dunaires sensibles à l'érosion. Actuellement, les initiatives de collecte d'informations et de mesures de l'effet des tempêtes sur les littoraux sableux se développent en France, comme le montre l'initiative du "Réseau Tempête d'Occitanie" dispositif fonctionnel à l'échelle de la région depuis 2011 (DE LA TORRE \& BALOUIN, 2012).

\subsection{Formules empiriques}

Lorsque les mesures et les observations de terrain n'existent pas ou sont trop lacunaires pour être interprétables, des formules empiriques peuvent être mise en œuvre. Toutefois ces approches nécessitent au minimum la connaissance d'un profil topographique perpendiculaire et représentatif de l'environnement étudié (plage, dune et arrière-dune) et des conditions hydrodynamiques locales en condition de tempête.

Aux Etats-Unis, la FEMA, Federal Emergency Management Agency, propose d'utiliser une règle simple et applicable sur les côtes de l'Atlantique et du Golfe du Mexique. Cette règle, basée sur une importante base de données d'observations, établi un volume de dune érodée en fonction de la période de retour (" $T$ " en années) associée au niveau d'eau extrême. Pour un profil perpendiculaire au trait de côte, la dune est érodée d'une aire " $A$ " (en $\left.\mathrm{m}^{2}\right)$ selon la formule suivante (Equation 2):

$A=8 \times T^{0.4}$

Pour un niveau d'eau centennal statique, l'érosion $A$ correspond à 540 pieds carrés $(540$ $\mathrm{SF}$ ) et donne son nom à la règle (FEMA, 1995). Si la surface de dune est supérieure à $50 \mathrm{~m}^{2}$ (équivalent à 540 pieds carrés), la dune est censée résister à la tempête.

Aux Pays-Bas, la méthode DUROS+ (DUne éROSion) est mise en œuvre pour estimer la résistance des dunes à l'érosion lors des tempêtes. Depuis 1986 (VELLINGA, 1986), cette méthode est régulièrement améliorée en utilisant notamment les résultats de modèles réduits physiques et de canaux à houle. Pour la plage après le pied de dune, le profil d'équilibre de forme parabolique est défini tel que $\mathrm{y}=\mathrm{f}(\mathrm{x})$ dans l'équation (3).

$$
\left(\frac{7.6}{H_{0 s}}\right) y=0.4714\left[\left(\frac{7.6}{H_{0 s}}\right)^{1.28}\left(\frac{12}{T_{p}}\right)^{0.45}\left(\frac{w_{s}}{0.0268}\right)^{0.56} x+18\right]^{0.5}-2
$$

Où $H_{0 s}(\mathrm{~m})$ correspond à la hauteur significative des vagues au large, Tp (s) est la période pic de vagues au large, $w_{s}(\mathrm{~m} / \mathrm{s})$ est la vitesse de chute des sédiments.

\subsection{Modélisation numérique}

Les modèles numériques sont des outils qui permettent une approche basée sur la reproduction des processus complexes agissant dans la zone littorale. Ainsi, le modèle SBeach (LARSON et al., 2004) estime l'extension de la zone du jet de rive et le 


\section{Thème 7 - Risques côtiers}

transport des sédiments pour déterminer l'évolution du profil de plage et de la dune lors d'une tempête.

On peut aussi citer le développement récent du modèle XBeach (ROELVINK et al., 2009) qui vise à reproduire les composantes parallèles et perpendiculaires à la côte des phénomènes hydro-sédimentaires lors des tempêtes, et notamment de l'énergie infragravitaire qui agit sur le jet de rive. Ce type de modèle se situe au niveau de l'état de l'art en recherche, il est toujours en phase de développement.

Sur des zones à enjeux, où la dynamique des dunes est sensible et complexe, ce type d'approche numérique peut permettre d'améliorer les résultats des méthodes empiriques d'estimation du recul dunaire lors d'évènements exceptionnels et de mettre en perspective les observations et mesures existantes.

\section{Applications et résultats}

\subsection{Observations et mesures}

En France, les mesures qui quantifient l'évolution plage-dune sous l'effet d'une tempête forte, très forte ou exceptionnelle sont encore rares. A ce titre, les informations collectées sur le terrain à la suite de la tempête Xynthia sur les côtes de Vendée et de Charente-Maritime ont permis d'identifier les zones de ruptures de cordon et d'estimer des reculs extrêmes pouvant atteindre $30 \mathrm{~m}$ très ponctuellement (GARCIN et al., 2011, figure 1).
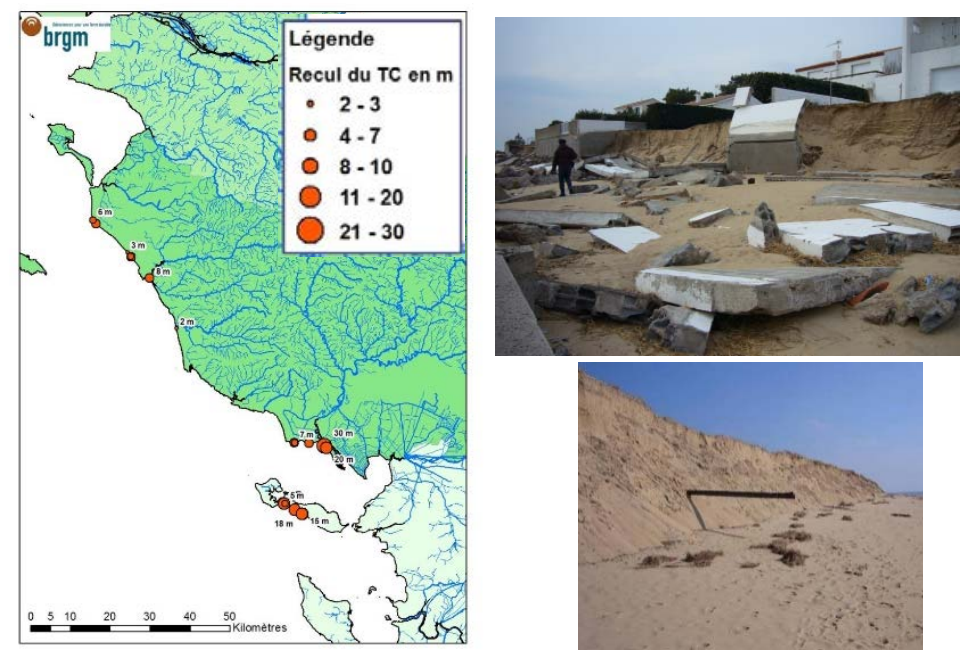

Figure 1. Reculs dunaires observés sur les côtes de Vendée après la tempête Xynthia.

Source : DESMAZES et al. (2014a).

Certains suivis annuels menés dans le cadre d'observatoires du littoral peuvent prendre en compte des évènements érosifs particuliers. Si les protocoles de suivi ne sont pas dédiés à la quantification des impacts spécifiques à un évènement, les mesures 


\section{XVèmes Journées Nationales Génie Côtier - Génie Civil \\ La Rochelle, 29 au 31 mai 2018}

disponibles peuvent cependant être utilisées en prenant des précautions et en intégrant une incertitude liée à l'évolution naturelle de la plage à l'échelle annuelle.

La figure 2 montre le recul annuel enregistré sur un profil particulier de Soulac-sur-Mer suivi depuis 2008 pour l'Observatoire de la côte Aquitaine (OCA). Entre 2013 et 2014, le recul est très fort et atteint $30 \mathrm{~m}$. Il est majoritairement associé au phénomène exceptionnel de succession de tempêtes de l'hiver 2013-2014 (BULTEAU et al., 2014).
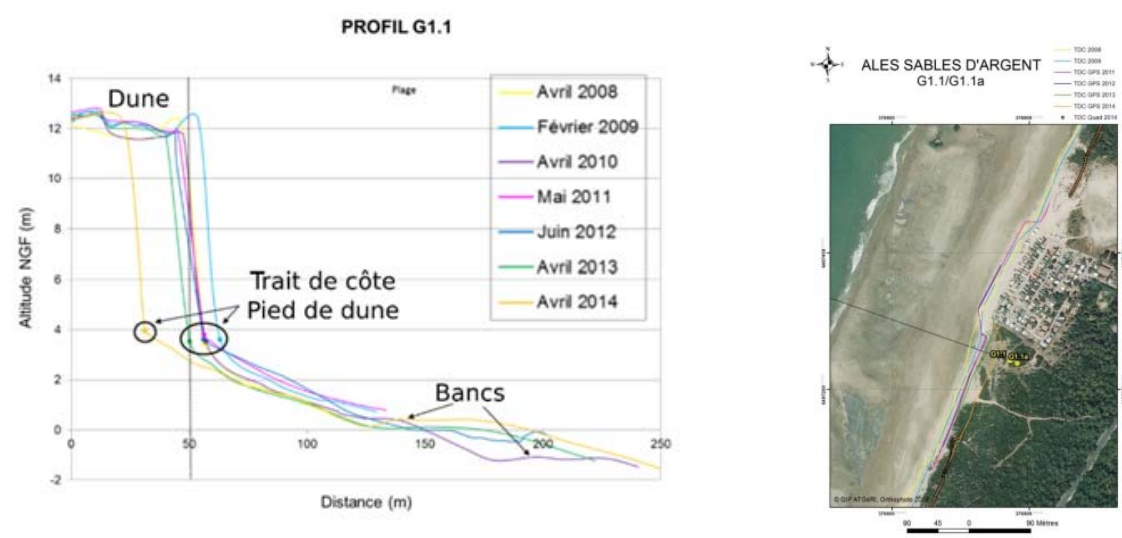

Figure 2. Mesures de l'évolution pluriannuelle du profil plage-dune et du trait de côte à Soulac-sur-Mer (OCA). Source : DESMAZES et al. (2014a).

Pour les secteurs régulièrement suivis mais présentant des enjeux très forts, il est possible de compenser la faible fréquence des mesures de terrain par l'installation de systèmes de vidéosurveillance dédiés au milieu littoral. Ces systèmes permettent le suivi en continu de différents indicateurs morphologiques, même durant les tempêtes (BALOUIN et al., 2012).

\subsection{Formules empiriques}

En France, il n'y a pas eu de développement de formulations empiriques dédiées à l'estimation du recul des dunes sous l'effet des tempêtes. La mise en place de ce type d'approche pour son application à des démarches sécuritaires comme les PPRL nécessiterait la constitution de bases de données dédiées aux différents types d'environnements dunaires et aux conditions de forçage rencontrées, afin de constituer des modèles paramétriques adaptés. Dans le cadre des études méthodologiques réalisées pour le compte de la DGPR, des tests d'utilisation des formules DUROS+ et FEMA ont été entreprises sur des sites Aquitains et Corses Ces sites, suivis régulièrement par des observatoires du littoral, ont permis une première évaluation des possibilités d'emploi de ces formules pour des cas français.

Pour des forçages dont les périodes de retour sont compris entre 1 et 10 ans, les résultats de la formule FEMA sont cohérents avec les observations de reculs maximaux observés, ce qui n'est pas le cas avec la formule DUROS+ qui semble surévaluer les valeurs. Pour des forçages dont les périodes de retour sont compris entre 50 et 100 ans, les valeurs 


\section{Thème 7 - Risques côtiers}

calculées sont cohérentes car elles dépassent les observations associées à des évènements de périodes de retour plus courtes. Dans certains cas (figure 3), les formules peuvent prédire la destruction de la dune, ce qui est une information majeure à ne pas négliger dans l'établissement de PPRL.
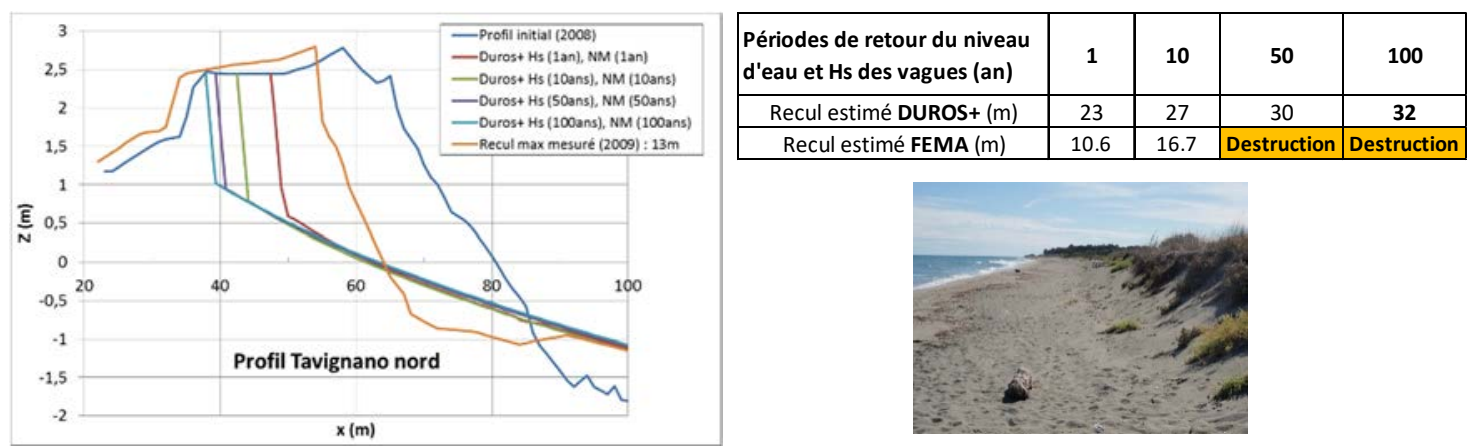

Figure 3. Tests des formules empiriques DUROS+ et FEMA sur la plage de Tavignano suivi par le Réseau d'Observation du Littoral Corse.

Dans le cadre d'études dédiées au PPRL et pour des secteurs à faibles enjeux, l'emploi de formules empiriques existantes (DUROS, FEMA, autres), même si elles n'ont pas été conçues spécifiquement pour l'environnement dunaire étudié, est envisageable pour permettre de discuter des informations historiques disponibles.

\subsection{Modélisation numérique}

XBeach est un modèle morphodynamique conçu pour reproduire l'évolution d'une plage et de la dune en 2D sous l'effet d'une tempête. Sa mise en œuvre nécessite des données morpho-bathymétriques et hydrodynamiques fiables et précises pour permettre le calibrage des paramètres et pour confronter les premiers résultats à des cas réels.

Dans un premier temps, le modèle a été testé pour des profils perpendiculaires simplifiés et des forçages représentatifs de différents milieux dunaires de métropole (Nord, Pertuis Charentais, Médoc, Landes, Occitanie, Corse orientale, DESMAZES et al., 2014b). Compte tenu du peu de données disponibles pour procéder à des calibrages, les reculs dunaires calculés apparaissent généralement sous-estimés, même pour des choix de forçages extrêmes. Seul l'exemple représentatif des lidos d'Occitanie a fourni des résultats significatifs en termes d'évolutions morphologiques du profil dunaire.

Dans un second temps, XBeach a été testé plus en détail afin d'évaluer sa capacité à reproduire les évolutions de la dune pour un cas réaliste de tempête. Le choix s'est porté sur la plage du Petit Travers, située sur le Lido de l'Or (Commune de Maugio-Carnon, 34). Ce secteur a fait l'objet d'un rechargement massif en 2007-2008 et d'un ambitieux programme de réaménagement de la dune. Lors de la tempête de novembre 2014 (figure 4), le front dunaire de la plage a été nettement érodé et des dégâts importants ont été constatés sur les aménagements d'accès à la plage. 


\section{XVèmes Journées Nationales Génie Côtier - Génie Civil \\ La Rochelle, 29 au 31 mai 2018}

L'analyse des résultats des différents tests de modélisation montre que, pour ce cas, le modèle présente une grande sensibilité à la morphologie initiale. L'érosion du front dunaire a pu être reproduite pour un profil initial de plage aérienne et sous-marine qui s'est érodée depuis les rechargements massifs opérés en 2007-2008. La qualité des jeux de données morphologiques disponibles apparait donc comme une contrainte forte pour l'utilisation de ce type d'outils numérique.
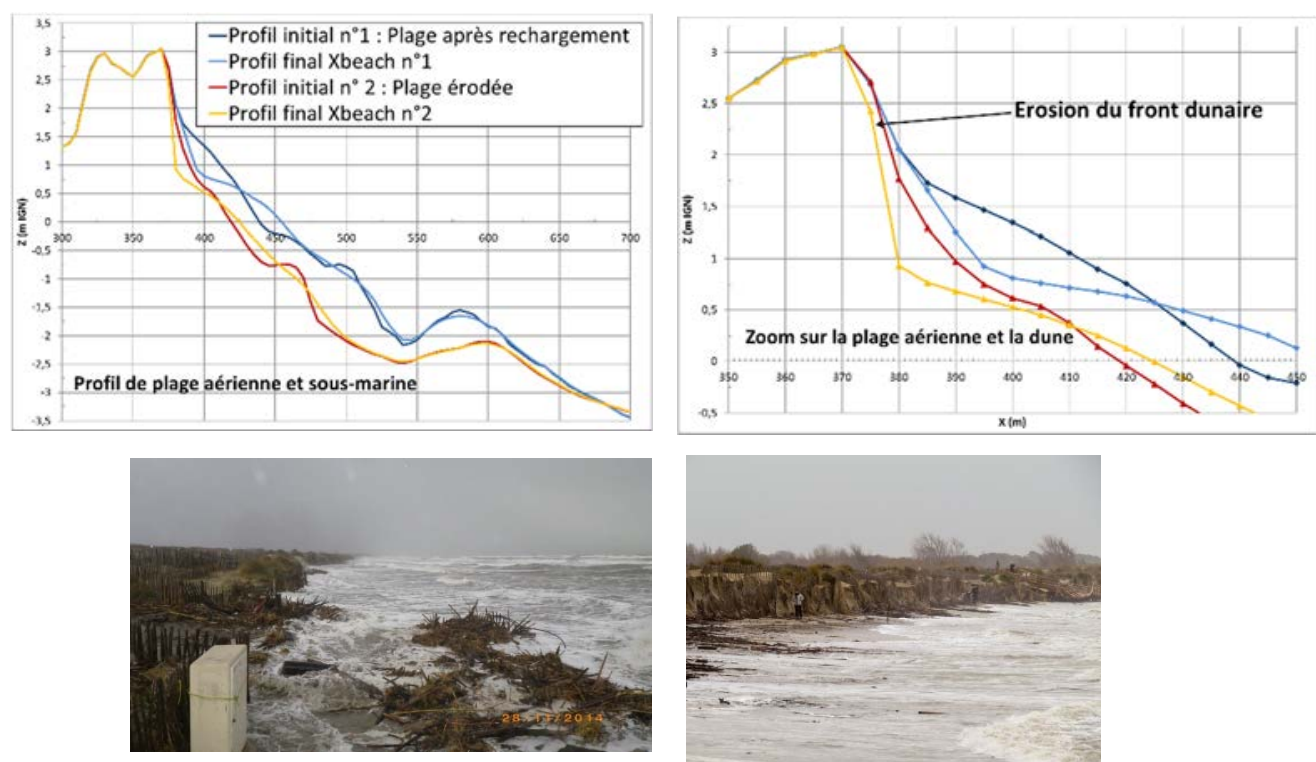

Figure 4. Erosion du front dunaire lors de la tempête du 28/11/2014 sur la plage du Petit travers (Réseau tempête LRO). Tests de modélisation XBeach de l'effet de la tempête. Source : DESMAZES et al. (2015).

\section{Conclusions}

Le recul instantané "Lmax" est un paramètre indispensable pour évaluer l'aléa de recul du trait de côté à 100 ans dans les Plans de Prévention des Risques Littoraux. Il doit permettre de prendre en compte des reculs du trait de côte exceptionnels qui ne sont pas correctement reproduits par les méthodes d'évaluation de l'aléa basées uniquement sur l'estimation d'un taux moyen.

Les travaux menés depuis 2013 par le BRGM pour le Ministère de l'Environnement, ont montré l'importance primordiale des observations post-tempêtes et des mesures d'évolutions morphologiques des dunes pour mieux évaluer ce paramètre Lmax. Pour les secteurs les moins connus ou à faibles enjeux, l'utilisation de formules empiriques issues de la bibliographie permet d'alimenter la discussion sur les hypothèses à retenir pour déterminer la valeur du Lmax. Les outils de modélisation numérique comme XBeach peuvent permettent de reproduire les évolutions morpho-sédimentaires de secteurs bien connus et parfaitement calibrés, à l'échelle de temps d'un évènement de tempête. Leur mise en œuvre est un atout pour les travaux dédiés aux secteurs à enjeux. 


\section{Thème 7 - Risques côtiers}

Toutefois, elle nécessite de mobiliser des jeux de données de grande qualité qui n'existent pas partout, mais qui mériteraient d'être développés pour les secteurs à forts enjeux particulièrement sensibles aux impacts des tempêtes.

\section{Références bibliographiques}

BUlteAU T., MUGICA J., MALlET C., GARNIER C., ROSEBERY D., et al. (2014). Évaluation de l'impact des tempêtes de l'hiver 2013-2014 sur la morphologie de la Côte Aquitaine. BRGM/RP-63797-FR. 49 p.

BALOUIN Y., DESBIENDRAS L., GERVAIS M., TESSON J. (2012). Dynamique à haute fréquence des barres d'avant-côte en milieu microtidal : suivi vidéo du Lido de Sète, Golfe du Lion. XII ${ }^{\text {èmes }}$ Journées Nationales Génie Côtier, Génie Civil, Cherbourg, pp 169-178. https://doi.org/10.5150/jngcgc.2012.019-B

DE LA TORRE Y., BALOUIN Y. (2012). Mise en œuvre opérationnelle du "Réseau Tempête" sur le littoral du Languedoc-Roussillon. BRGM/RP-60694-FR. 97 p., 15 ill., 5 ann.

DESMAZES F., MULLER H., GARCIN M., BELON R., BAUDOIN V. (2014a) Méthodologie de détermination du recul maximal des dunes pour les événements extrêmes. BRGM/RP-63157-FR. 131 p

DESMAZES F., MULLER H., NICOLAE LERMA A. (2014b). Méthodologie de détermination du recul maximal des dunes pour les événements extrêmes. Phase 2/A. BRGM/RP-64184-FR. 58 p.

DESMAZES F., MULlER H., NICOLAE LERMA A., BULTEAU T. (2015). Méthodologie de détermination et d'estimation du recul maximal des dunes lors d'évènements extrêmes. Phase 2 - Année 2. BRGM/RP-65439-FR, 94 p.

DGPR (2014). Guide méthodologique : Plan de prévention des risques littoraux. 169 p. FEMA (1995). Guidelines and specifications for wave elevation determination and $v$ zone mapping. $160 \mathrm{p}$.

GARCIN M., et al. (2011). Base de données d'observation des effets de la tempête Xynthia sur le littoral. BRGM/RP-59395- FR. 23 p.

LARSON M., WISE R.A., KRAUS N.C. (2004). Coastal overwash: Part 2, Upgrade to SBEACH. Coastal and Hydraulics Laboratory, $21 \mathrm{p}$.

ROELVINK D., RENIERS A., VAN DONGEREN A., VAN THIEL DE VRIES J., MCCALL R., LESCINSKI J. (2009). Modelling storm impacts on beaches, dunes and barrier islands. Coastal Engineering, Vol. 56(11-12), pp 1133-1152. https://doi.org/10.1016/j.coastaleng.2009.08.006

VELLINGA P. (1986) Beach and Dune Erosion During Storm Surges. PhD, 169 p.

VAN GENT M.R.A., VAN THIEL DE VRIES J.S.M., COEVELD E.M., DE VROEG J.H., VAN DE GRAAFF J. (2008). Large-scale dune erosion tests to study the influence of wave periods. Coastal Engineering, Vol. 55(12), pp 1041-1051. https://doi.org/10.1016/j.coastaleng.2008.04.003 